\title{
CuZnSOD and MnSOD inhibit metabolic stress-induced necrosis and multicellular tumour spheroid growth
}

\author{
SU YEON LEE ${ }^{1}$, HYUN MIN JEON ${ }^{1}$, CHO HEE KIM ${ }^{1}$, EUI KYONG JEONG ${ }^{1}$, MIN KYUNG JU ${ }^{1}$, SONG YI PARK ${ }^{1}$, \\ SEUNG YOUN JUNG ${ }^{1}$, YUNG JIN KIM ${ }^{1}$, SUNG-CHUL LIM ${ }^{2,3}$, SONG IY HAN ${ }^{2}$ and HO SUNG KANG ${ }^{1}$ \\ ${ }^{1}$ Department of Molecular Biology, College of Natural Sciences, Pusan National University, \\ Pusan 609-735; ${ }^{2}$ Research Center for Resistant Cells and ${ }^{3}$ Department of Pathology, \\ College of Medicine, Chosun University, Gwangju 501-759, Korea
}

Received January 14,2010; Accepted March 23, 2010

DOI: 10.3892/ijo_00000667

\begin{abstract}
CuZnSOD and MnSOD have been shown to exert tumour suppressive activities; however, their exact molecular mechanism is still unclear. We investigated the molecular mechanism underlying the tumour suppressive activities of CuZnSOD and MnSOD using multicellular tumour spheroid (MTS), an in vitro tumour model. Overexpression of CuZnSOD and MnSOD significantly suppressed the growth of A549 and MCF-7 MTS, supporting a critical role(s) of reactive oxygen species (ROS) in tumour growth. In solid tumours, ROS is produced by metabolic stress due to insufficient oxygen and glucose supply and induces necrosis that is known to promote tumour progression by releasing the proinflammatory cytokine HMGB1. We observed that CuZnSOD and MnSOD overexpression prevents metabolic stress-induced necrosis and HMGB1 release by inhibiting mitochondrial ROS and intracellular $\mathrm{O}_{2}^{-}$production in response to glucose depletion in two dimensional cell culture. CuZnSOD and MnSOD overexpression also significantly repressed the occurrence of necrosis that was observed during MTS culture. In human tumour tissues including lung pulmonary adenocarcinoma, $\mathrm{CuZnSOD}$ and MnSOD expression was detected in the paranecrotic region that was identified by the expression of a hypoxic marker carbonic anhydrase (CA) IX. These results suggest that CuZnSOD and MnSOD may suppress tumour growth through inhibiting metabolic stress-induced necrosis and HMGB1 release via inhibiting metabolic stress-induced mitochondrial ROS production.
\end{abstract}

\section{Introduction}

Reactive oxygen species (ROS) such as $\mathrm{O}_{2}^{-}, \mathrm{H}_{2} \mathrm{O}_{2}$ and $\mathrm{OH}^{-}$are involved in a diverse of cell regulation and play an important

Correspondence to: Dr Ho Sung Kang, Department of Molecular Biology, College of Natural Sciences, Pusan National University, Pusan 609-735, Korea

E-mail: hspkang@pusan.ac.kr

Key words: MnSOD, CuZnSOD, necrosis, multicellular tumour spheroid growth role(s) in tumour development (1-4). While excess ROS levels are toxic to cancer cells, chronic ROS initiate malignant transformation either by causing mutagenic DNA alterations and oncogene activation and/or tumour suppressor gene inactivation, or by regulating oncogenic signaling pathways such as PTEN and modulating transcription factors such as HIF-1 $\alpha$ (1-3). ROS could be produced by the mitochondrial electron transport chain, and by the action of enzymatic systems. Under normal conditions, they are maintained at low levels by several anti-oxidants enzymes including cytosolic CuZn superoxide dismutase (CuZnSOD), mitochondrial MnSOD, glutathione peroxidase (GPx), and catalase (CAT). Superoxide dismutases (SOD) catalyze the conversion of $\mathrm{O}_{2}^{-}$ to $\mathrm{H}_{2} \mathrm{O}_{2}$ that is then detoxified to $\mathrm{H}_{2} \mathrm{O}$ by CAT and GPx. Impairment of the antioxidant system renders cells vulnerable to the effects of ROS, including DNA mutation and protein modifications which contribute to tumourigenesis (1-3).

MnSOD and CuZnSOD have been demonstrated to function as tumour suppressors in many cancer cell lines (5-7). For instance, overexpression of MnSOD protein inhibits growth in a wide variety of cancer types including human melanoma cells, SV40-transformed lung fibroblasts, glioma cells, pancreatic cancer cells, and androgen-independent prostate cancer cells (8-12). It has been also shown that a reduction in MnSOD activity results in a much higher incidence of cancer (13). In addition, overexpression of CuZnSOD decreases not only cell growth but also in vivo xenograft growth compared to controls $(14,15)$. CuZnSOD deficiency is also reported to lead to persistent oxidative damage and hepatocarcinogenesis (16). The levels of MnSOD and CuZnSOD are known to be low in tumour tissues when compared with normal tissues, supporting the role of MnSOD and $\mathrm{CuZnSOD}$ as tumour suppressors $(6,7)$. Reduced expression of MnSOD is thought to be due to either mutations in the promoter of the gene and in the coding sequence, or hypermethylation of $\mathrm{CpG}$ islands in the promoter regions to silence gene expression (17-19). Tumour suppressive activity of MnSOD has been demonstrated to be linked to its abilities to inhibit HIF-1 protein accumulation and VEGF expression $(20,21)$ and to upregulate maspin, one of the serpin family of protease inhibitors that function as a tumour-suppressor (22). 
However, the molecular mechanism underlying the tumour suppressive activities of CuZnSOD and MnSOD remains unclear.

When a solid tumour is growing, its center becomes distant from surrounding blood vessels leading to the appearance of hypoxic and glucose depleted (OGD) areas that are common features of solid tumours (23-25). Cancer cells become adapted to these stresses, allowing them to survive and grow under OGD conditions, contributing to the malignant phenotype and to aggressive tumour progression; otherwise, they exhibit necrotic cell death to form the necrotic core. Necrosis is induced by ROS that is produced by metabolic stress due to insufficient oxygen and glucose supply. Unlike apoptosis where the cell content remains packed in the apoptotic bodies that are removed by marcrophages, and thereby inflammation does not occur, necrosis is characterized by the cell membrane rupture, and the release of cytosolic constituents including HMGB1 into the extracellular space causing a massive inflammatory response (26-28). HMGB1 could act as a cytokine by signaling via the receptor for advanced glycated end-products (RAGE) and via members of the Toll-like receptor family, thereby initiating inflammatory responses. Recently, HMGB1 has been shown to promote tumour progression $(29,30)$. In this study, we investigated the molecular mechanism underlying the tumour suppressive activities of CuZnSOD and MnSOD using multicellular tumour spheroid (MTS), an in vitro tumour model. Here we show that CuZnSOD and MnSOD may suppress the growth of A549 and MCF-7 MTS by inhibiting metabolic stress-induced necrosis and HMGB1 release via inhibiting metabolic stressinduced mitochondrial ROS production.

\section{Materials and methods}

Cell culture and glucose depletion (GD). Human lung adenocarcinoma cell line A549 and breast cancer cell line MCF-7 were obtained from American Type Culture Collection and grown in RPMI-1640 media (Gibco BRL) supplemented with $10 \%(\mathrm{v} / \mathrm{v})$ heat-inactivated fetal bovine serum (FBS, Gibco $\mathrm{BRL}$ ) and $1 \%$ penicillin-streptomycin (PS, Gibco BRL) in a $37^{\circ} \mathrm{C}$ humidified incubator with $5 \% \mathrm{CO}_{2}$. For glucose depletion (GD), cells were gently rinsed twice with glucosefree RPMI-1640 and incubated in GD medium (glucose-free RPMI-1640 medium (Gibco BRL) containing 10\% dialyzed and heat-inactivated FBS and 1\% PS).

Multicellular tumour spheroid (MTS) culture. A549 and MCF-7 cells were cultured to $80 \%$ confluence and seeded into $1.2 \%$ agarose-coated 96 -well plates at a density of 4,000 cells and 400 cells per well, respectively, and cultured. Each well contained $200 \mu 1$ of tissue culture medium, and the spheroids were fed every other day by carefully aspirating $100 \mu 1$ of spent medium and replacing with the same quantity of fresh medium. To determine the MTS growth, diameters of spheroids were measured every day.

SDS-PAGE and Western blot analysis. Sodium dodecyl sulfatepolyacrylamide gel electrophoresis on $12 \%$ gels and Western blotting with antibodies to CuZnSOD, MnSOD (Santa Cruz, CA), ERK1/2 (Cell Signaling, MA), $\alpha$-tubulin (Biogenex,
CA), and HMGB1 (BD Pharmingen) was performed as described previously $(31,32)$.

Measurement of intracellular ROS. To determine production of intracellular $\mathrm{H}_{2} \mathrm{O}_{2}, \mathrm{O}_{2}^{-}$, and mitochondrial ROS, the cells were loaded with 2,7-dichlorofluorescin diacetate (DCFH-DA, Molecular Probes, $50 \mu \mathrm{M}$ ), dihydroethidium (HE, Molecular Probes, $10 \mu \mathrm{M}$ ) or reduced form of MitoTracker Red CM$\mathrm{H}_{2} \mathrm{Xros}$ (a mitochondrial specific probes that fluoresce upon oxidation, thereby is used to detect mitochondrial ROS, Molecular Probes, $50 \mathrm{nM}$ ) for 30 min under growth condition and then rinsed and soaked in phenol red-free RPMI. DCFHDA and HE fluorescence was excited at $488 \mathrm{~nm}$ using an argon laser, and evoked emission was filtered with a 515-nm (DCFH-DA) or 515-605-nm (HE) band-pass filter and Mito Tracker Red CM- $\mathrm{H}_{2}$ Xros was excited at $579 \mathrm{~nm}$, and emitted at $599 \mathrm{~nm}$ and the cells were observed under a laser-scaning confocal microscope (LSM510, Carl Zeiss).

Hoechst 33342 (HO)/propidium iodide (PI) staining. Cells were incubated either with $1 \mu \mathrm{g} / \mathrm{ml} \mathrm{HO}$ and $5 \mu \mathrm{g} / \mathrm{ml}$ PI at $37^{\circ} \mathrm{C}, 5 \% \mathrm{CO}_{2}$ for $15 \mathrm{~min}$ in the dark $(31,32)$. Both floating and attached cells were collected by centrifugation of the medium and trypsinization, respectively. The pooled cell pellets were immediately fixed in $3.7 \%$ formaldehyde, washed with phosphate-buffered saline (PBS), resuspended, and a fraction of the suspension was centrifuged in a cytospinner (Thermo Shandon, Shandon Inc.). The slides were washed in PBS to remove excessive dye, air dried, mounted in FluroGard Antifade, and then examined under a fluorescence microscope (340/425 nm (HO), and 580/630 nm (PI) (DM5000, Leica, Germany).

$H M G B 1$ release assay. Cell culture medium was collected at the indicated time points and cells and debris were removed by centrifugation at $2400 \mathrm{x} \mathrm{g}$ for $20 \mathrm{~min}$ at $4^{\circ} \mathrm{C}$ and the supernatant was filtered through Centricon YM-100 (Millipore) to clear the samples from cell debris. Then, samples were concentrated 15-fold with Centricon YM-30 and analyzed by Western blotting with antibody to polyclonal anti-HMGB1 antibodies (BD Pharmingen).

CuZnSOD and MnSOD transfection. The gene encoding for MnSOD was amplified from genomic DNA by the polymerase chain reaction (PCR) with a specific primer set and cloned into the eukaryotic expression vector pcDNA3.1 (Invitrogen). pEGFP-CuZnSOD (provided by B.J. Pak, Pusan National University) was constructed by inserting the CuZnSOD open reading frame into plasmid pEGFP (Clontech, Mountain View, CA). Plasmids pcDNA3.1-MnSOD and pEGFP-CuZnSOD were transfected to A549 cells using lipofectamine 2000 (Gibco BRL) and selected with geneticin (G418, Gibco BRL) according to the manufacturer's instructions.

Immunohistochemistry. Immunohistochemistry (IHC) was performed on 4- $\mu \mathrm{m}$ sections of formalin-fixed, paraffinembedded tissues. Sections were deparaffinized in xylene and graded alcohol. Antigen retrieval was performed by autoclaving for $15 \mathrm{~min}$. After incubation with blocking solution for $30 \mathrm{~min}$, sections were incubated with antibodies to CuZnSOD, MnSOD 
A
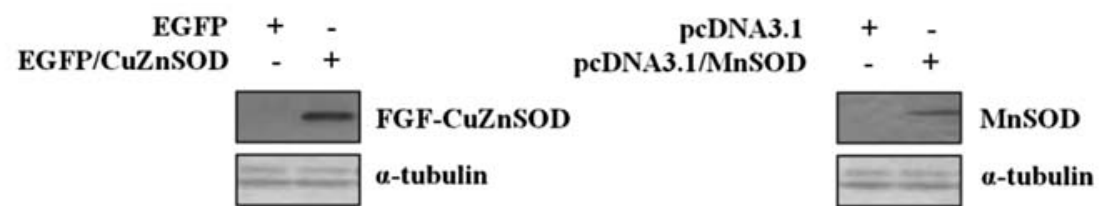

B
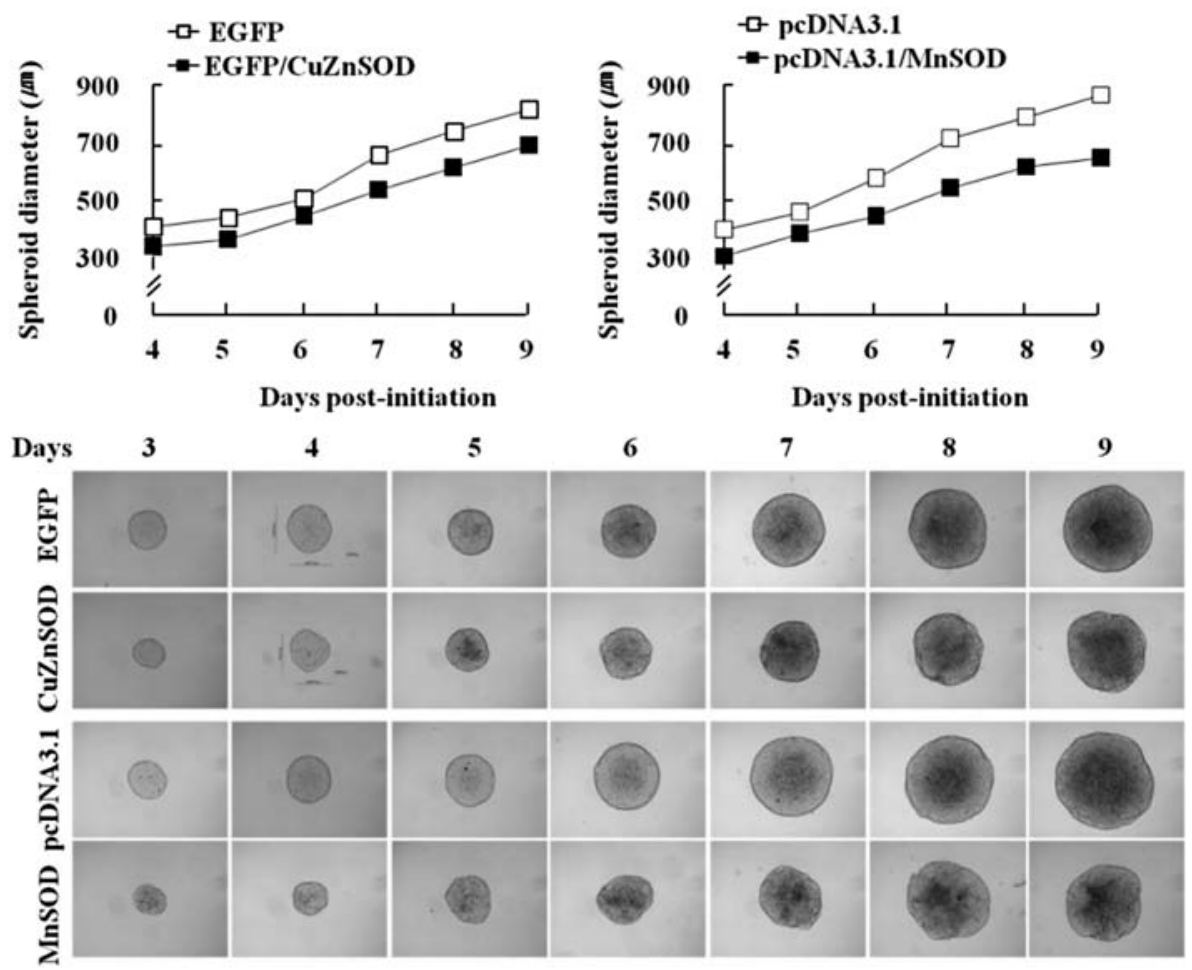

C
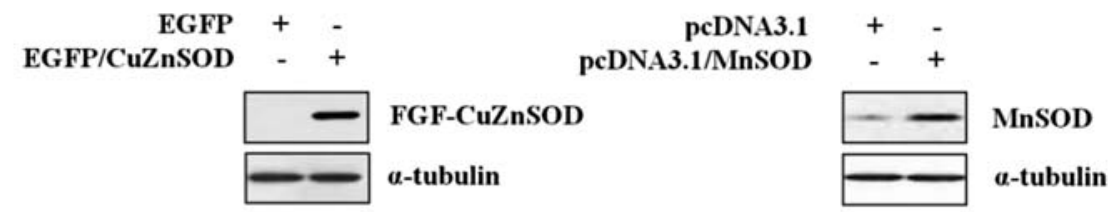

D
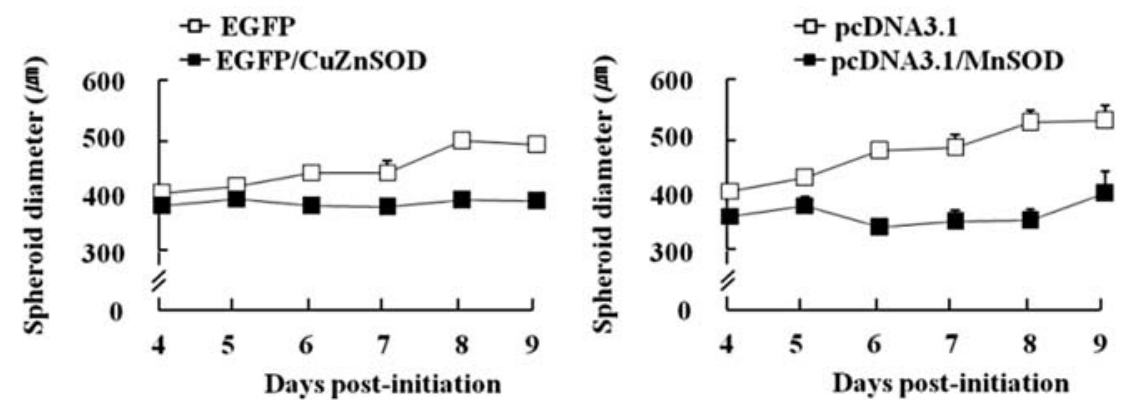

Figure 1. Overexpression of CuZnSOD and MnSOD significantly suppresses the growth of MTS. CuZnSOD- and MnSOD-overexpressing MCF-7 (A and B) and A549 (C and D) cells were cultured to $80 \%$ confluence and seeded into $1.2 \%$ agarose-coated 96 -well plates at a density of 400 cells and 4,000 cells per well, respectively, and cultured for up to 9 days. To determine the MTS growth, diameters of 5 spheroids were measured every day.

(Santa Cruz), and phospho-ERK2 (Cell Signaling) for $1 \mathrm{~h}$, and biotinylated secondary antibody for $20 \mathrm{~min}$ and then with streptavidin horseradish peroxidase (HRP) for $10 \mathrm{~min}$. Staining was carried out with diaminobenzidine (DAB) chromogen and counterstaining with hematoxylin.

\section{Results and Discusion}

CuZnSOD and MnSOD suppress multicellular tumour spheroid (MTS) growth. CuZnSOD and MnSOD have been shown to exert tumour suppressive activities; however, their exact molecular mechanism remains unclear. We examined the effects of CuZnSOD and MnSOD overexpression on the tumour cell growth using MTS, an in vitro model of solid tumours $(33,34)$. For this purpose, we used breast cancer cell line MCF-7 and human lung adenocarcinoma cell line A549. MCF-7 and A549 cells were seeded into 1.2\% agarose-coated 96-well plates at a density of 400 cells and 4,000 cells per well, respectively, and the morphological features of spheroids were observed by light microscopy (Fig. 1). When seeded 
A

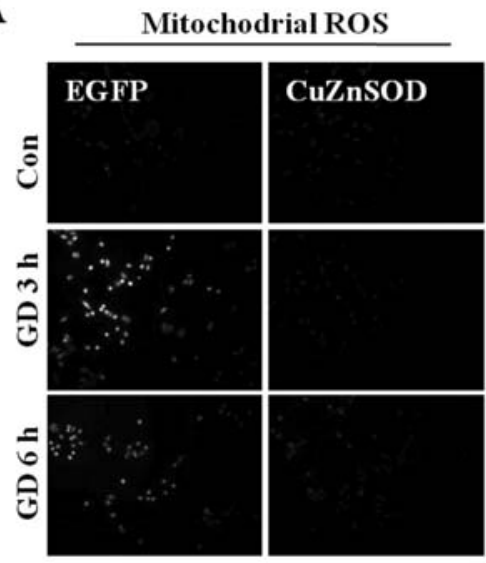

B

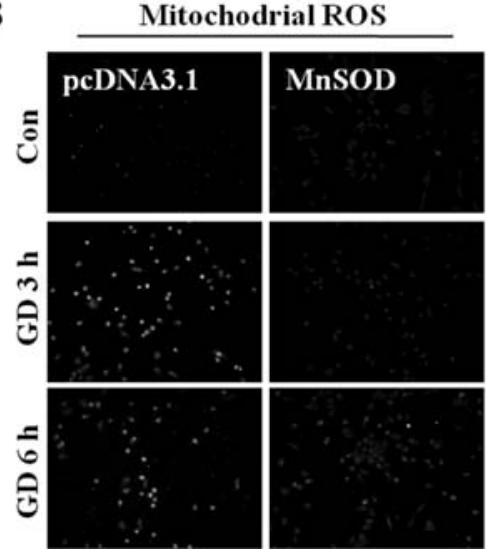

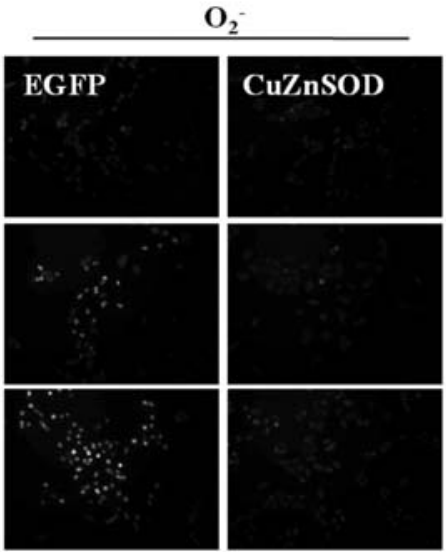
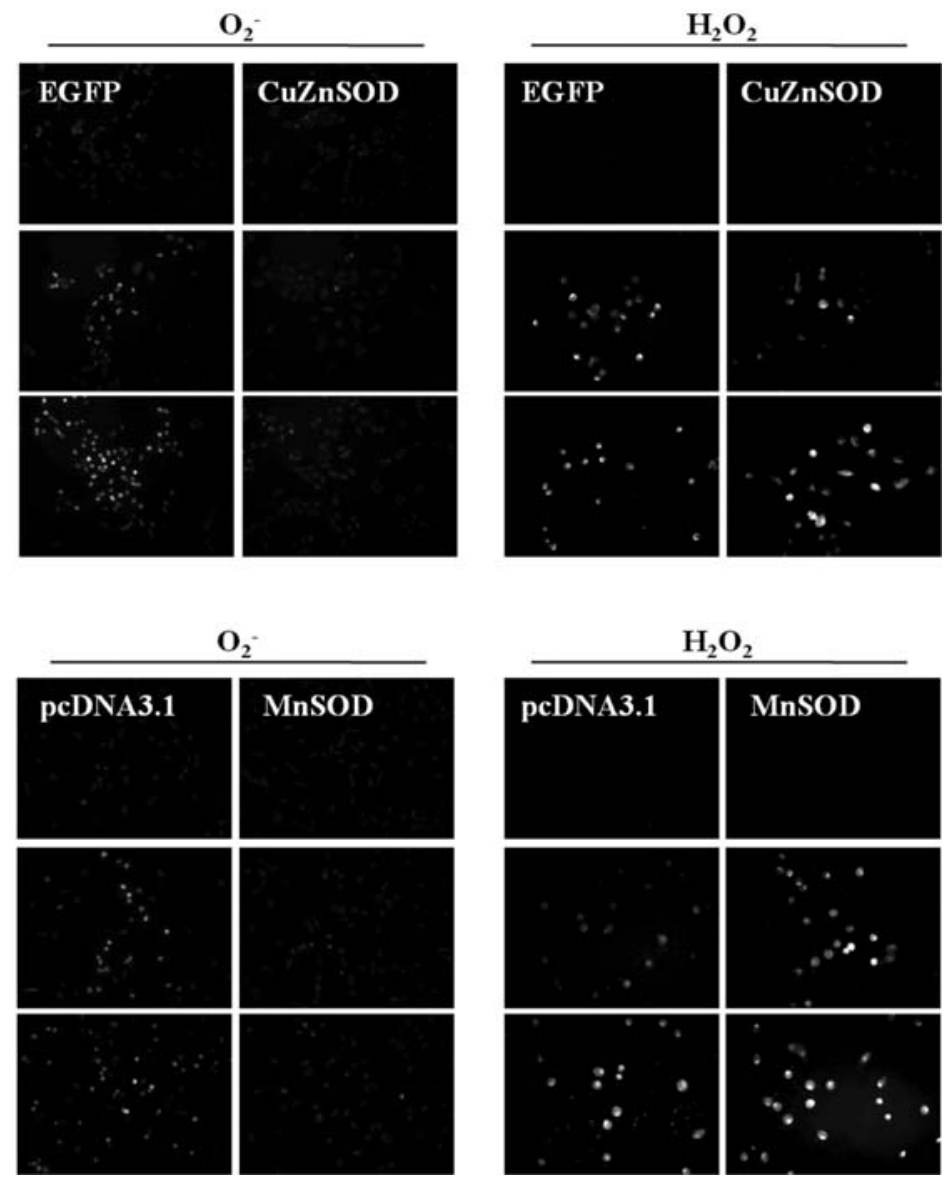

Figure 2. The effects of CuZnSOD and MnSOD on GD-induced ROS production. CuZnSOD (A) and MnSOD (B) overexpressing A549 cells were cultured in normal growth medium or GD medium for 3 and $6 \mathrm{~h}$. The cells were treated with DCFH-DA, HE, and MitoTracker RedCM- ${ }_{2} \mathrm{XROS}$ and observed under a confocal microscope (x200, CarlZeiss, LSM510) to detect intracellular mitochondrial ROS, superoxide $\left(\mathrm{O}_{2}^{-}\right)$, and $\mathrm{H}_{2} \mathrm{O}_{2}$.

in these non-adhesive conditions, the cells aggregated and formed multicellular spheroids. MCF-7 formed a compact spherical shape of spheroids (Fig. 1), whereas A549 cells generated slightly loose aggregates (data not shown). Such difference in compact MTS formation is likely due to their differential expression of surface adhesion molecules, such as E-cadherin or N-cadherin. We found that CuZnSOD and MnSOD suppress the growth of both MCF-7 and A549 MTS and after 9-day culture, the difference was marked (Fig. 1). These results support a critical role(s) of ROS in tumour growth.

CUZnSOD and MnSOD inhibit metabolic stress-induced necrosis and HMGB1 release. In solid tumours, ROS is produced by metabolic stress due to insufficient oxygen and glucose supply. As demonstrated previously $(31,32)$, GD, one of metabolic stress, could induce intracellular $\mathrm{H}_{2} \mathrm{O}_{2}$ and $\mathrm{O}_{2}^{-}$production in A549 cells (Fig. 2). The source of ROS production appeared to be the mitochondria (Fig. 2). Thus, we investigated the effects of CuZnSOD and MnSOD on GD-induced ROS production. As shown in Fig. 2, CuZnSOD and MnSOD overexpression prevented production of mitochondrial ROS and intracellular $\mathrm{O}_{2}{ }^{-}$in response to GD, but not intracellular $\mathrm{H}_{2} \mathrm{O}_{2}$. CuZnSOD and MnSOD are known to scavenge $\mathrm{O}_{2}{ }^{-}$but to promote $\mathrm{H}_{2} \mathrm{O}_{2}$ formation. Then, we examined the effects of CuZnSOD and MnSOD overexpression on GD-induced necrosis. Necrotic and apoptotic cell death were determined by $\mathrm{HO} / \mathrm{PI}$ double staining methods. In HO/PI double staining method, DNAbinding dye $\mathrm{HO}$ is known to cross the plasma membrane of all cells, whether they are damaged or not, causing a blue fluorescence of their nuclei, while PI only penetrates cells with damaged membranes and leads to nuclear fluorescence. Thus, intact blue nuclei, condensed/fragmented blue nuclei, condensed/fragmented pink nuclei, and intact pink nuclei were considered viable, early apoptotic, late apoptotic (secondary necrotic), and necrotic cells, respectively. GD increased the population of the cells that had intact pink nuclei (HO/PI staining, Fig. 3A and D). CuZnSOD (Fig. 3A and B) and MnSOD (Fig. 3D and E) overexpression significantly reduced the population of PI-positive cells. GDinduced HMGB1 release was also suppressed by overexpression of CuZnSOD and MnSOD (Fig. 3C and F), indicating that $\mathrm{CuZnSOD}$ and MnSOD exert the anti-necrotic acivity to prevent HMGB1 release possibly by their ability to scavenge ROS. Previously, we showed that NAC (a general anti-oxidant) and catalase (a specific antioxidant for $\mathrm{H}_{2} \mathrm{O}_{2}$ ) prevent necrotic cell death and switched the cell death mode to apoptosis (31). However, overexpression of $\mathrm{CuZnSOD}$ and MnSOD blocked GD-induced necrotic cell death but did not switch the cell death mode to apoptosis. Similarly, tiron (a scavenger of $\mathrm{O}_{2}^{-}$) was shown to block GD-induced 
A

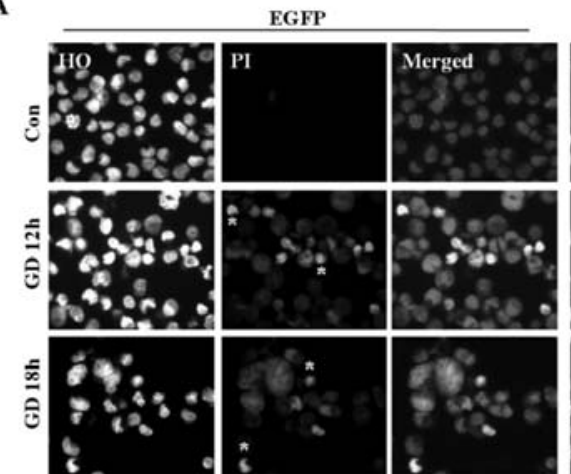

CuZnSOD

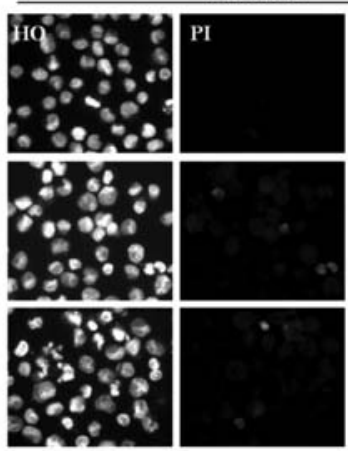

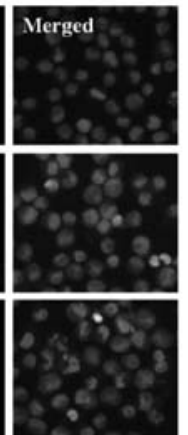
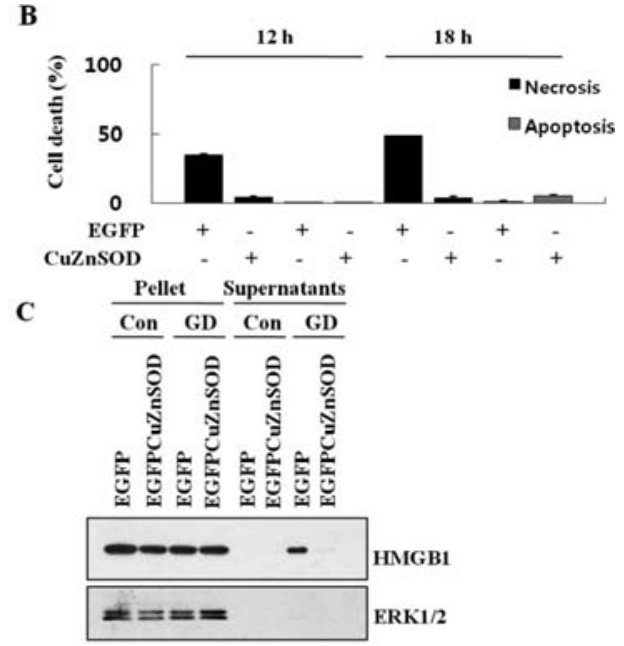

D
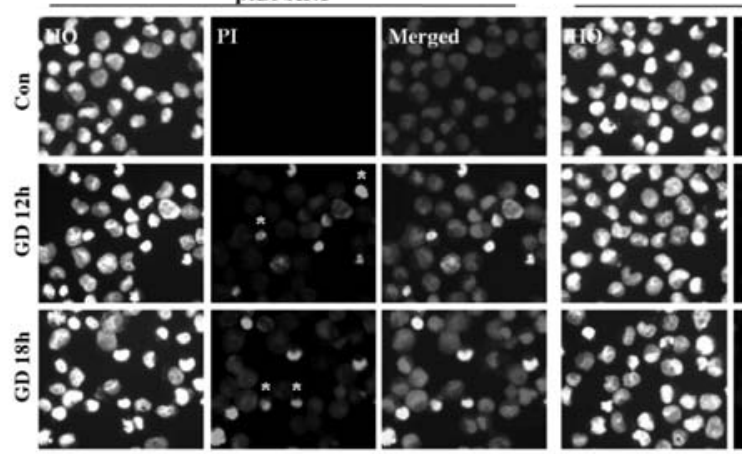

MnSOD
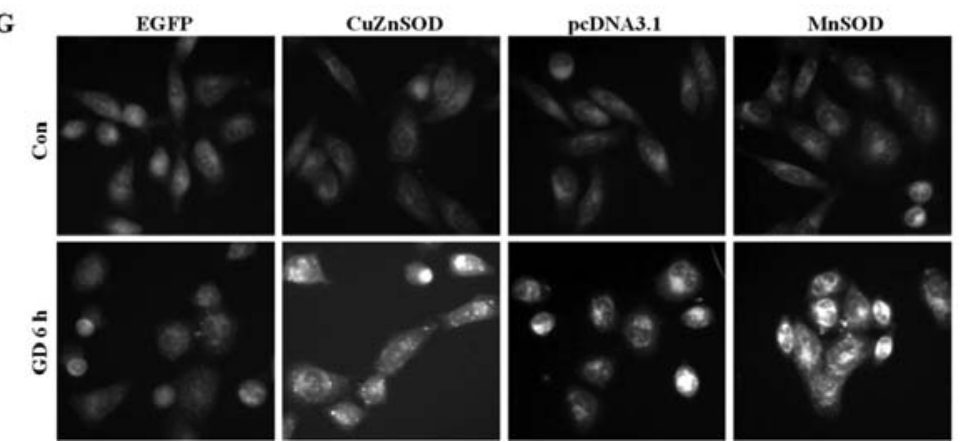

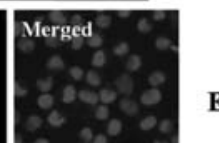

$\mathbf{E}$
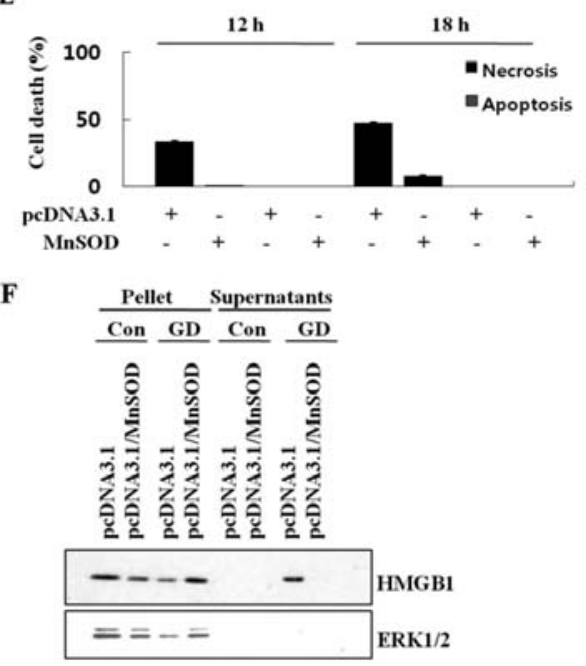

Figure 3. The effects of CuZnSOD and MnSOD overexpression on metabolic stress-induced necrosis and HMGB1 release in A549 cells. CuZnSOD (A and B) and MnSOD (D and E) overexpressing A549 cells were cultured in normal growth medium or GD medium for 12 and $18 \mathrm{~h}$ and then stained with HO/PI and observed with fluorescence microscopy (low panel) and apoptotic and necrotic cells were scored (upper panel). Results (500-800 cells in each group) are expressed as the means \pm SEM from three independent experiments. ${ }^{*}$ Necrotizing cells. CuZnSOD (C) and MnSOD (E) overexpressing A549 cells were treated with GD medium for $12 \mathrm{~h}$ and both the cell medium (supernatants) and the cells (pellets) were prepared as described in Materials and methods and analyzed by Western blotting with antibody to HMGB1 and ERK1/2. (G) CuZnSOD and MnSOD prevent necrosis and switched the cell death mode to autophagy. CuZnSOD- and MnSOD-overexpressing A549 cells were cultured in normal growth medium or GD medium for $6 \mathrm{~h}$ and stainined with MDC.

necrotic cell death without switching the cell death mode to apoptosis (31). SOD over-expression may lead to changes in the superoxide/hydrogen peroxide balance. Since SOD and tiron are known to scavenge $\mathrm{O}_{2}{ }^{-}$but to promote $\mathrm{H}_{2} \mathrm{O}_{2}$ formation, $\mathrm{O}_{2}^{-}$seems to act as a major ROS to induced necrotic cell death and excess $\mathrm{H}_{2} \mathrm{O}_{2}$ may prevent the molecular mechanism underlying apoptotic cell death. Recently, $\mathrm{H}_{2} \mathrm{O}_{2}$, has been shown to be essential for autophagosome formation and autophagic degradation under starvation conditions and the cysteine protease HsAtg 4 is a direct target for oxidation by $\mathrm{H}_{2} \mathrm{O}_{2}$ (35). Thus, we examined the possibility whether $\mathrm{CuZnSOD}$ and MnSOD overexpression may switch metabolic stress-induced necrosis to autophagy. The formation of autophagic vacuoles was assessed by staining with MDC, which accumulates in acidic cell compartments enriched in lipids and leads to a punctate staining pattern when autophagy is stimulated. As shown in Fig. 3G, CuZnSOD and MnSOD overexpressing cells showed apparent accumulation of MDC in the cytoplasmic vacuoles when exposed to $\mathrm{GD}$, indicating that increases in $\mathrm{H}_{2} \mathrm{O}_{2}$ levels in response to $\mathrm{CuZnSOD}$ and $\mathrm{MnSOD}$ may switch the metabolic stress-induced necrosis to autophagy.

CuZnSOD and MnSOD repress the occurrence of necrosis during MTS culture. As observed within tumours, continued spheroid growth leads to the formation of necrotic core due to microenvironmental stresses including deprivation of oxygen and nutrients. Necrotic cell death was observed at day 8 in MCF-7 MTS culture and at day 7 in A549 MTS culture. We examined the effects of CuZnSOD and MnSOD 
A
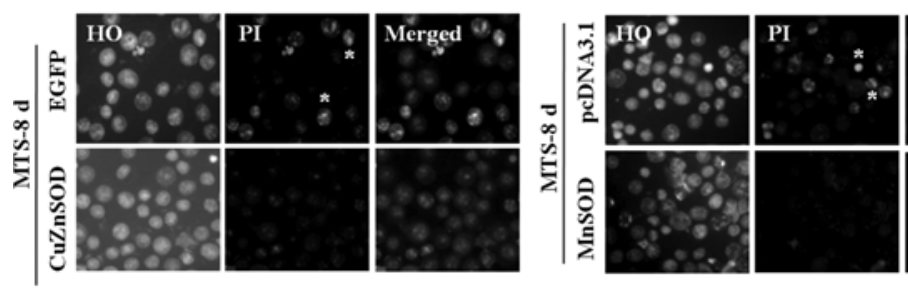

C
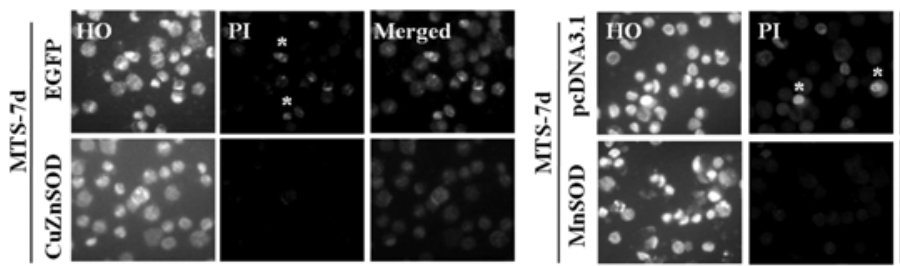

B
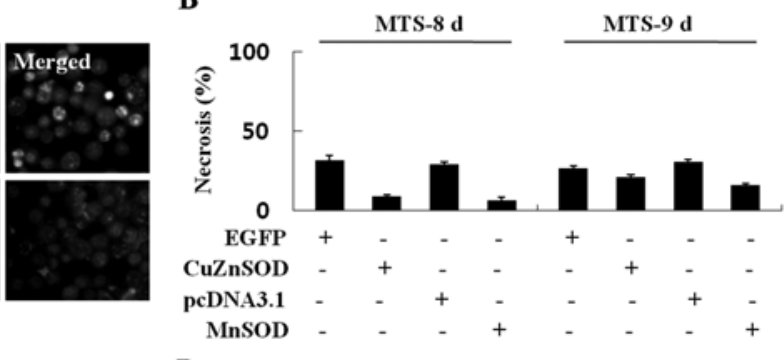

D
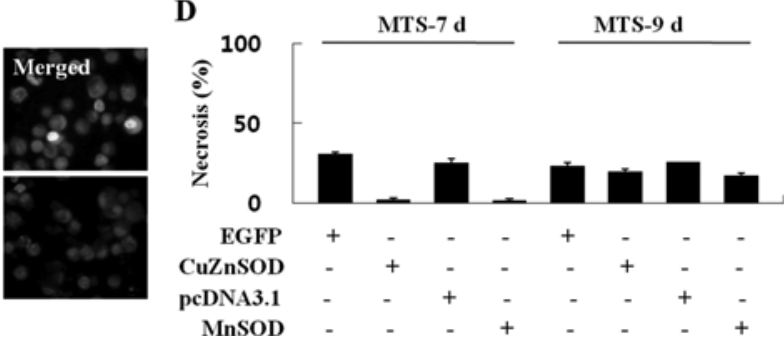

Figure 4. Overexpression of CuZnSOD and MnSOD suppresses necrotic cell death in MTS. CuZnSOD- and MnSOD-overexpressing MCF-7 (A and B) and A549 (C and D) cells were seeded into 1.2\% agarose-coated 96-well plates at a density of 400 cells and 4,000 cells per well, respectively, and cultured for 7 and 9 days. Then the cells were dissociated and stained with HO/PI and observed with fluorescence microscopy (A and C) and apoptotic and necrotic cells were scored (B and D). Results (500-800 cells in each group) are expressed as the means \pm SEM from three independent experiments. "Necrotizing cells.
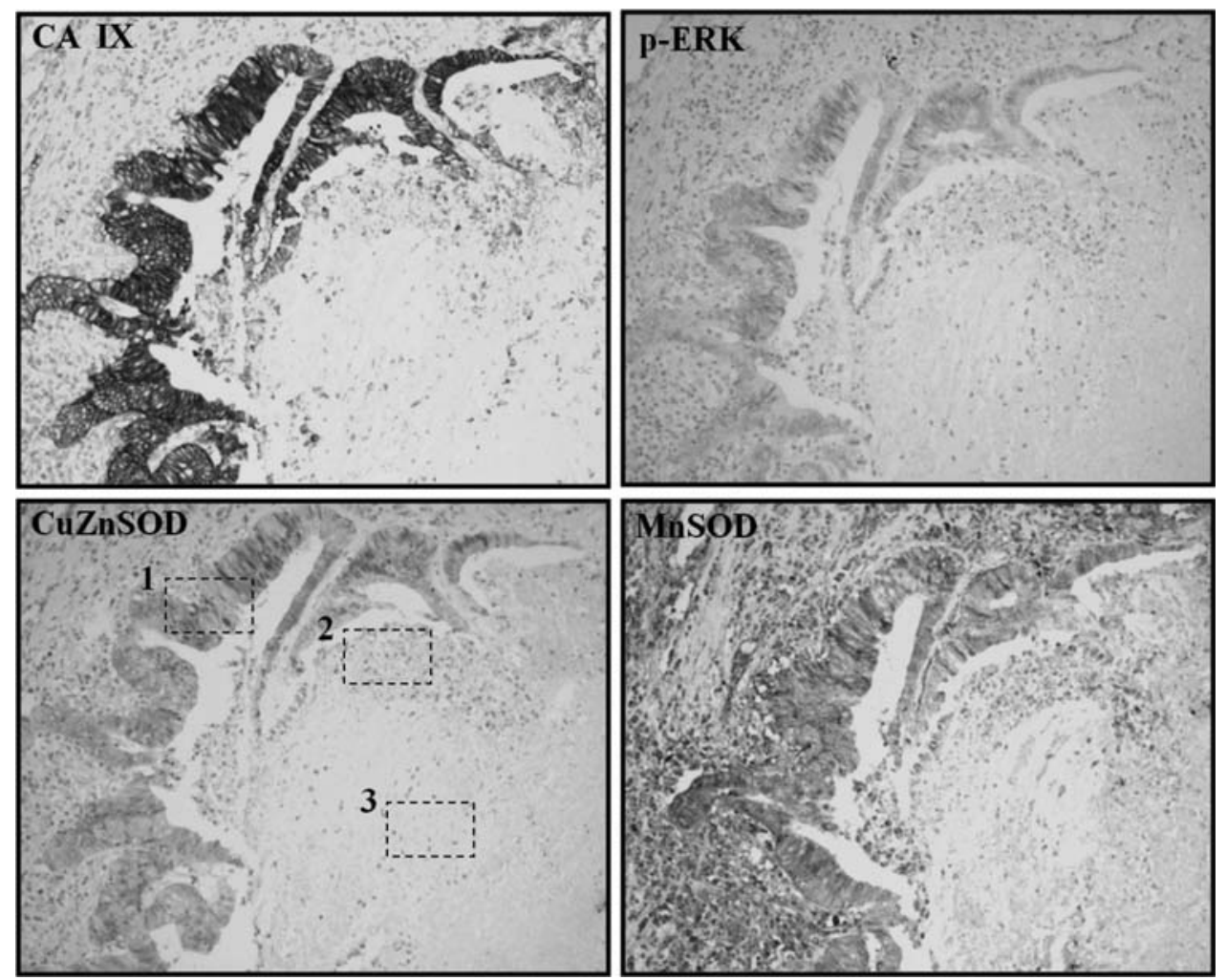

Figure 5. Immunohistochemcal observation of CA IX, CuZnSOD, MnSOD, and phospho-ERK2 in lung pulmonary adenocarcinoma. 1, The para-necrotic region that was identified by the expression of a hypoxic marker CA IX; 2 and 3 , the necrotic region.

on the necrotic cell death observed in MTS. We found that overexpression of CuZnSOD and MnSOD supresses the occurrence of necrotic cell death in MTS (Fig. 4). A prominent anti-necrotic activity of CuZnSOD and MnSOD was observed at day 8 in MCF-7 MTS culture and at day 7 in A549 MTS culture, while it was less at day 9 in MCF-7 and
A549 MTS culture. Thus, CuZnSOD and MnSOD overexpression appeared to significantly retard the occurrence of necrotic cell death in MTS.

CuZnSOD and MnSOD expression in solid tumours. We examined $\mathrm{CuZnSOD}$ and MnSOD expression in solid tumours 
including lung pulmonary adenocarcinoma, metastatic colonic carcinoma in liver, and metastatic lung pulmonary adenocarcinoma in skin by immunohistochemistry. In lung pulmonary adenocarcinoma, CuZnSOD and MnSOD expression was detected in the para-necrotic region that was identified by the expression of a hypoxic marker CA IX (Fig. 5). Similar results were obtained in metastatic colonic carcinoma in liver and metastatic lung pulmonary adenocarcinoma in skin (data nort shown), supporting the anti-necrotic activity of CuZnSOD and MnSOD. Active form of ERK that has been shown to exert an anti-necrotic activity (31) also colocalized with CA IX and SOD (Fig. 5). Although the levels of MnSOD and CuZnSOD has been reported to be low in tumour tissues when compared with normal tissues $(6,7)$, an increased MnSOD expression or activity was also reported in invasive cancers including gastric, brain astrocytic and colorectal carcinomas compared to non-malignant tissue that are associated with either metastasis, invasiveness or poor prognosis (36-41). Here, we report that CuZnSOD and MnSOD expression was detected in the para-necrotic region in solid tumours. CA IX is a hypoxia-induced enzyme that has many biologically important functions, including its role in cell adhesion and invasion and its expression is known to be significantly higher in MnSOD-positive tumours (42). The reason for MnSOD and CuznSOD expression in the paranecrotic region is unclear. Although necrosis is known to exhibit the tumour promoting and angiogenic activities by releasing HMGB1, excessive necrosis and inflammation can lead to tumour cytotoxicity and regression through high level infiltration of monocytes and/or neutrophil. Thus, MnSOD and $\mathrm{CuZnSOD}$ expression in the para-necrotic region may suppress the development of excessive inflammation that could prevent tumour progression. MnSOD and CuZnSOD have been reported as tumour suppressors in many cancer cell lines (5-7). Our results suggest that the ability of MnSOD and CuZnSOD to suppress metabolic stress-induced necrosis and HMGB1 release may contribute to their tumour suppressive activities, thereby preventing malignant and aggressive tumour progression.

\section{Acknowledgements}

This work was supported by the National Research Foundation of Korea (NRF) grant funded by the Korea government (MEST) (2009-0059210), and a grant of the Korea Healthcare technology R\&D Project, Ministry for Health, Welfare, Korea (A080303).

\section{References}

1. Fruehauf JP and Meyskens FL Jr: Reactive oxygen species: a breath of life or death? Clin Cancer Res 13: 789-794, 2007.

2. Feig DI, Reid TM and Loeb LA: Reactive oxygen species in tumorigenesis. Cancer Res 54: S1890-S1894, 1994.

3. Breimer LH: Molecular mechanisms of oxygen radical carcinogenesis and mutagenesis: the role of DNA base damage. Mol Carcinog 3: 188-197, 1990.

4. Wu WS: The signaling mechanism of ROS in tumor progression. Cancer Metastasis Rev 25: 695-705, 2006.

5. St Clair D, Zhao Y, Chaiswing L and Oberley T: Modulation of skin tumorigenesis by SOD. Biomed Pharmacother 59: 209-214, 2005.

6. Kinnula VL and Crapo JD: Superoxide dismutases in malignant cells and human tumors. Free Radic Biol Med 36: 718-744, 2004.
7. Oberley LW: Mechanism of the tumor suppressive effect of MnSOD overexpression. Biomed Pharmacother 59: 143-148, 2005.

8. Church SL, Grant JW, Ridnour LA, Oberley LW, Swanson PE, Meltzer PS and Trent JM: Increased manganese superoxide dismutase expression suppresses the malignant phenotype of human melanoma cells. Proc Natl Acad Sci USA 90: 3113-3117, 1993.

9. Yan T, Oberley LW, Zhong W and St Clair DK: Manganesecontaining superoxide dismutase overexpression causes phenotypic reversion in SV40-transformed human lung fibroblasts. Cancer Res 56: 2864-2871, 1996.

10. Zhong W, Oberley LW, Oberley TD and St Clair DK: Suppression of the malignant phenotype of human glioma cells by overexpression of manganese superoxide dismutase. Oncogene 14: 481-490, 1997.

11. Weydert C, Roling B, Liu J, Hinkhouse MM, Ritchie JM, Oberley LW and Cullen JJ: Suppression of the malignant phenotype in human pancreatic cancer cells by the overexpression of manganese superoxide dismutase. Mol Cancer Ther 2: 361-369, 2003.

12. Venkataraman S, Jiang X, Weydert C, Zhang Y, Zhang HJ, Goswami PC, Ritchie JM, Oberley LW and Buettner GR: Manganese superoxide dismutase overexpression inhibits the growth of androgen-independent prostate cancer cells. Oncogene 24: 77-89, 2005.

13. Van Remmen H, Ikeno Y, Hamilton M, Pahlavani M, Wolf N, Thorpe SR, Alderson NL, Baynes JW, Epstein CJ, Huang TT, Nelson J, Strong R and Richardson A: Life-long reduction in MnSOD activity results in increased DNA damage and higher incidence of cancer but does not accelerate aging. Physiol Genomics 16: 29-37, 2003.

14. Zhang Y, Zhao W, Zhang HJ, Domann FE and Oberley LW: Overexpression of copper zinc superoxide dismutase suppresses human glioma cell growth. Cancer Res 62: 1205-1212, 2002.

15. Weydert CJ, Waugh TA, Ritchie JM, Iyer KS, Smith JL, Li L, Spitz DR and Oberley LW: Overexpression of manganese or copper-zinc superoxide dismutase inhibits breast cancer growth. Free Radic Biol Med 41: 226-237, 2006.

16. Elchuri S, Oberley TD, Qi W, Eisenstein RS, Jackson Roberts L, van Remmen H, Epstein CJ and Huang TT: CuZnSOD deficiency leads to persistent and widespread oxidative damage and hepatocarcinogenesis later in life. Oncogene 24: 367-380, 2005.

17. Xu Y, Krishnan A, Wan XS, Majima H, Yeh CC, Ludewig G, Kasarskis EJ and St Clair DK: Mutations in the promoter reveal a cause for the reduced expression of the human manganese superoxide dismutase gene in cancer cells. Oncogene 18: 93-102, 1999.

18. Huang Y, He T and Domann FE: Decreased expression of manganese superoxide dismutase in transformed cells is associated with increased cytosine methylation of the SOD2 gene. DNA Cell Biol 18: 643-652, 1999.

19. Hernandez-Saavedra D and McCord JM: Paradoxical effects of thiol reagents on Jurkat cells and a new thiol-sensitive mutant form of human mitochondrial superoxide dismutase. Cancer Res 63: 159-163, 2003.

20. Wang M, Kirk JS, Venkataraman S, Domann FE, Zhang HJ, Schafer FQ, Flanagan SW, Weydert CJ, Spitz DR, Buettner GR and Oberley LW: Manganese superoxide dismutase suppresses hypoxic induction of hypoxia-inducible factor-1alpha and vascular endothelial growth factor. Oncogene 24: 8154-8166, 2005.

21. Kaewpila S, Venkataraman S, Buettner GR and Oberley LW: Manganese superoxide dismutase modulates hypoxia-inducible factor-1 alpha induction via superoxide. Cancer Res 68: 2781-2788, 2008.

22. Li JJ, Colburn NH and Oberley LW: Maspin gene expression in tumor suppression induced by overexpressing manganesecontaining superoxide dismutase cDNA in human breast cancer cells. Carcinogenesis 19: 833-839, 1998.

23. Guppy M: The hypoxic core: a possible answer to the cancer paradox. Biochem Biophys Res Commun 299: 676-680, 2002.

24. Gatenby RA and Gillies RJ: Why do cancers have high aerobic glycolysis? Nat Rev Cancer 4: 891-899, 2004.

25. Harris AL: Hypoxia - a key regulatory factor in tumour growth. Nat Rev Cancer 2: 38-47, 2002.

26. Kanduc D, Mittelman A, Serpico R, Sinigaglia E, Sinha AA, Natale C, Santacroce R, Di Corcia MG, Lucchese A, Dini L, Pani P, Santacroce S, Simone S, Bucci R and Farber E: Cell death: apoptosis versus necrosis (review). Int J Oncol 21: 165-170, 2002. 
27. Zong WX and Thompson CB: Necrotic death as a cell fate. Genes Dev 20: 1-15, 2006.

28. Golstein P and Kroemer G: Cell death by necrosis: towards a molecular definition. Trends Biochem Sci 32: 37-43, 2007.

29. Vakkila $J$ and Lotze MT: Inflammation and necrosis promote tumour growth. Nat Rev Immunol 4: 641-648, 2004.

30. Lotze MT and Tracey KJ: High-mobility group box 1 protein (HMGB1): nuclear weapon in the immune arsenal. Nat Rev Immunol 5: 331-342, 2005.

31. Kim CH, Han SI, Lee SY, Youk HS, Moon JY, Duong HQ, Park MJ, Joo YM, Park HG, Kim YJ, Yoo MA, Lim SC and Kang HS: Protein kinase C-ERK1/2 signal pathway switches glucose depletion-induced necrosis to apoptosis by regulating superoxide dismutases and suppressing reactive oxygen species production in A549 lung cancer cells. J Cell Physiol 211: 371-385, 2007.

32. Lim SC, Choi JE, Kim CH, Duong HQ, Jeong GA, Kang HS and Han SI: Ethyl pyruvate induces necrosis-to-apoptosis switch and inhibits high mobility group box protein 1 release in A549 lung adenocarcinoma cells. Int J Mol Med 20: 187-192, 2007.

33. Horning JL, Sahoo SK, Vijayaraghavalu S, Dimitrijevic S, Vasir JK, Jain TK, Panda AK and Labhasetwar V: 3-D tumor model for in vitro evaluation of anticancer drugs. Mol Pharm 5: 849-862, 2008.

34. Ivascu A and Kubbies M: Diversity of cell-mediated adhesions in breast cancer spheroids. Int J Oncol 31: 1403-1413, 2007.

35. Scherz-Shouval R, Shvets E, Fass E, Shorer H, Gil L and Elazar Z: Reactive oxygen species are essential for autophagy and specifically regulate the activity of Atg4. EMBO J 26: 1749-1760, 2007.
36. Izutani R, Asano S, Imano M, Kuroda D, Kato M and Ohyanagi H: Expression of manganese superoxide dismutase in esophageal and gastric cancers. J Gastroenterol 33: 816-822, 1998.

37. Toh Y, Kuninaka S, Oshiro T, Ikeda Y, Nakashima H, Baba H, Kohnoe S, Okamura T, Mori M and Sugimachi K: Overexpression of manganese superoxide dismutase mRNA may correlate with aggressiveness in gastric and colorectal adenocarcinomas. Int $\mathbf{J}$ Oncol 17: 107-112, 2000.

38. Nozoe T, Honda M, Inutsuka S, Yasuda M and Korenaga D: Significance of immunohistochemical expression of manganese superoxide dismutase as a marker of malignant potential in colorectal carcinoma. Oncol Rep 10: 39-43, 2003.

39. Ho JC, Zheng S, Comhair SA, Farver C and Erzurum SC: Differential expression of manganese superoxide dismutase and catalase in lung cancer. Cancer Res 61: 8578-8585, 2001.

40. Skrzycki M, Scibior D, Podsiad M and Czeczot H: Activity and protein level of CuZnSOD and MnSOD in benign and malignant liver tumors. Clin Biochem 41: 91-96, 2008.

41. Svensk AM, Soini Y, Paakko P, Hiravikoski P and Kinnula VL: Differential expression of superoxide dismutases in lung cancer. Am J Clin Pathol 122: 395-404, 2004.

42. Jarvela S, Parkkila S, Bragge H, Kahkonen M, Parkkila AK, Soini Y, Pastorekova S, Pastorek J and Haapasalo H: Carbonic anhydrase IX in oligodendroglial brain tumors. BMC Cancer 8: $1,2008$. 